\title{
LA ETNOGRAFÍA COMO HERRAMIENTA DE INNOVACIÓN DOCENTE. Primeros pasos de una investigación sobre turismo y ocio
}

\author{
José A. MANSILLA; Yadur GONZALEZ; Sheila SANCHEZ BERGARA; Claudio MILANO \\ Universitat de Lleida \\ joseamansilla@hotmail.com,yngonzalez@ostelea.com,ssanchezb@ostelea.com, \\ claudiomilanouab@gmail.com
}

THE ETHNOGRAPHY AS A TEACHING INNOVATION TOOL. First steps of an investigation on tourism and leisure

Resumen: La etnografía ha sido considerada, tradicionalmente, como uno de los distintivos más importantes de ciencias sociales como la antropología y la sociología. Aunque en origen se aplicó para estudiar pequeñas comunidades de las denominadas "sociedades primitivas", pronto mostró, paralelamente, su potencialidad en contextos urbanos, tal y como la Escuela de Chicago se encargó de demostrar. El paso del tiempo ha permitido que continúe mostrando su enorme plasticidad como herramienta de estudio social, alcanzando nuevos campos de aplicación, como la educación. Basada en la experiencia llevada a cabo en torno al Grado de Turismo y Ocio de la Escuela Universitaria de Turismo Ostelea, centro adscrito de la Universitat de Lleida (UdL), la presente investigación muestra los resultados de una investigación en marcha que persigue analizar la capacidad de la metodología etnográfica para convertirse en una herramienta eficaz en procesos de innovación docente.

Abstract: Traditionally, ethnography has been considered as one of the most important elements of distinction in social sciences such as anthropology and sociology. Although originally was applied to study small communities of the so-called "primitive societies", it soon showed, in parallel, its potential in urban contexts, as the Chicago School demonstrated. Time has allowed it to continue showing its enormous plasticity as a social tool, reaching new fields of application, such as education. Based on the experience carried out around the Bachelor in Tourism and Leisure of the Ostelea School of Tourism and Hospitality, University of Lleida (UdL), this research shows the results of an ongoing process that seeks to analyze the capacity of the ethnographic methodology as an effective tool in teaching innovation processes.

Palabras clave: Etnografía. Turismo. Docencia. Metodología Ethnography. Tourism. Teaching. Methodology 


\section{Breve introducción}

Lejos quedaron ya los tiempos en los que la educación, formal y no formal, en sus distintos niveles quedaba reducida a una serie de charlas magistrales impartidas por un profesorado considerado como poseedor de cualidades casi demiúrgicas. La evolución de la sociedad, así como los cambios, sucesivos y considerables, de la educación, la han situado, hoy en día, en la línea de una formación amplia que incluye aspectos vinculados con la identidad y la concepción de la realidad, destacando la necesidad de difundir el conocimiento, pero también incidiendo en una valoración ética sobre el proceso educativo mismo. Esta visión y espíritu crítico aparece, incluso, recogido en la legislación educativa. Así, la gran apuesta por una educación acorde a los tiempos de los primeros Gobiernos españoles de la democracia, la Ley Orgánica de Ordenación General del Sistema Educativo (Ley 1/1990 de 3 de octubre), ya señalaba que la educación debería ir "dirigida al desarrollo de su capacidad para ejercer, de manera crítica y en una sociedad axiológicamente plural, la libertad, la tolerancia y la solidaridad". La misma Ley, en su preámbulo, insistía en la necesidad de considerar a la educación como un proceso que acompañará a los ciudadanos a lo largo de toda su vida, adaptándose de forma continua a los tiempos que le tocara vivir. En lo que respecta a la educación superior universitaria, esto también quedaba explícito en la última normativa aprobada, la Ley Orgánica de Universidades (6/2001 de 21 de diciembre), donde se subrayaba, en su exposición de motivos, la importancia de la innovación docente "en las formas de generación y transmisión de conocimiento"2.

En este sentido, el proceso de innovación educativa que viene desarrollando la Escuela Universitaria de Turismo Ostelea, centro adscrito a la Universitat de Lleida (UdL), se enmarca en la línea propuesta de mejora continua del proceso de enseñanza-aprendizaje y en su alineación con una visión crítica, a la vez que constructiva, de la actividad docente. De este modo, entre los elementos a los que se les ha prestado especial atención podemos encontrar los contextos y los métodos utilizados para tal fin en el Grado en Turismo y Ocio, y entre los que destaca el uso de la metodología etnográfica. Estudios previos sobre el uso de la etnografía en contextos educativos (Moreno y Daza, 2015; Maturana y Garzón, 2015) argumentan su validez metodológica en dinámicas didáctico-pedagógicas que tienen como objetivo la investigación social, al tiempo que destacan su pertinencia para el estudio de los propios entornos educativos.

En este artículo se persigue presentar los resultados iniciales de una investigación en marcha sobre el uso de técnicas propias del método etnográfico -herramientas tradicionalmente vinculadas a los estudios antropológicos y sociológicos- en la educación universitaria. Para ello, se analiza esta técnica en el proceso de creación de conocimientos y adquisición de competencias por parte de estudiantes del Grado en Turismo y Ocio. Para la consecución de este objetivo se presentan y discuten los resultados de incorporar la práctica etnográfica a dos contextos específicos de la ciudad de Barcelona (España), localidad donde se ubica la Escuela Universitaria. Por un lado, el barrio del Raval en el Distrito de Ciutat Vella, centro actual de ocio nocturno y contexto urbano sometido tradicionalmente a grandes intervenciones urbanísticas y, por otro lado, el entorno de la Cárcel Modelo, en el Distrito del Eixample, prisión clausurada recientemente y cuyo espacio liberado albergará, entre otros elementos, un museo dedicado a su memoria. Los motivos de su elección, así como las características espaciales y sociales de los mismos, serán introducidos más adelante. Tras una presentación inicial de tipo teórico, se expondrán sendos relatos etnográficos sobre experiencias concretas de trabajo etnográfico con estudiantes de Grado. Para finalizar, se presentaran unas conclusiones provisionales, donde se mostrará a la etnografía como una herramienta que contribuiría a una mejor comprensión de categorías, contextos, prácticas y significados de las experiencias de ocio y turismo, aspectos que se alcanzan tanto mediante el uso de contextos educativos diferentes al aula, como con el mantenimiento por parte del estudiante de un rol activo en el proceso de aprendizaje.

\section{A qué nos referimos cuando hablamos de etnografía}

Existen diferentes métodos que permiten, por un lado, evaluar los procesos de aprendizaje llevados a cabo en el ámbito educativo y, por otro, entender las problemáticas del entorno donde el docente se encuentra ejerciendo su labor. La etnografía ha sido, de forma tradicional, entendida como una metodología vinculada a las ciencias sociales. Sin embargo, también se ha manifestado como una significativa herramienta

\footnotetext{
1 Para más información ver: https://www.boe.es/buscar/doc.php?id=BOE-A-1990-24172. Con posterioridad a esta Ley ha existido un amplio desarrollo legislativo de sus preceptos, así como otras leyes educativas que han modificado sustancialmente sus principios y preceptos principales. La última, la Ley Orgánica para la Mejora de la Calidad Educativa (LOMCE), o Ley Wert, ha sido ampliamente criticada. Para más información ver: “¿Por qué la LOMCE es tan polémica? Los profesores responden al Gobierno”, El Confidencial, 09/06/2013, o "Los diez puntos más conflictivos de la LOMCE", abc, 16/04/2016. Pese a todo, elementos tales como la necesidad de innovar de forma continua el desarrollo de los modos de enseñanza, han permanecido inalterados. 2 Para más información ver: https://www.boe.es/buscar/act.php?id=BOE-A-2001-24515
} 
vinculada a la innovación docente. Su planteamiento admite no sólo identificar problemas, sino entender el contexto donde el docente lleva a cabo su labor, aportando alternativas de solución conforme a las características de la realidad en el que está inmerso. Su planteamiento permite la observación e interpretación de las identidades, costumbres y tradiciones de las comunidades, sea cual sea su localización.

Es ampliamente reconocido que los cánones de la antropología consideran a Bronisław Malinowski (1884-1942) como el padre de la etnografía. Para éste, dicha herramienta consistía en una serie de técnicas utilizadas para la comprensión de la interacción de los sujetos con sus contextos; profundizando en el estudio de los procesos sociales para un mayor conocimiento de la relación del ser humano con sus pares, su entorno natural y su cosmogonía. Así "el objetivo final [...] es comprender el punto de vista del nativo, su relación con la vida, entender su visión del mundo" (Malinowski, 1922: 24-25). De este modo, la etnografía consiste en la descripción de la formas de vida de los seres humanos a través de una observación lo más aguda y amplia posible con el objetivo de lograr un estudio asaz detallado de la cultura de una comunidad, esto es, el análisis de los modos de vida de un grupo de individuos, de lo que hacen, de su comportamiento, sus interacciones y el conjunto de creencias, valores, estímulos y aspiraciones según momentos y circunstancias (Mauss, 1967; Aguirre Baztán, 1995 y Nolla, 1997). En este sentido, la etnografía ofrece la oportunidad a las investigaciones de apartarse del enfoque tradicional positivista de tipo cuantitativo y avanzar hacia otro enfoque de carácter descriptivo o explicativo.

Como metodología, está integrada por diferentes etapas. El proceso se inicia desde el momento en que la idea se está gestando, periodo tan variable que lo mismo puede llevar unos días hasta varios meses. De ahí se pasa al encuentro con la comunidad donde se realizará la indagación mediante la observación, la descripción o recogida de datos, el análisis y la interpretación de los hechos, tarea ardua esta última ya que en la investigación cualitativa el estudioso se encuentra con datos de características muy variadas, de gran volumen y únicas a la vez. Todas estas circunstancias llevan a que el análisis, en ocasiones, se torne difícil y complejo, de ahí que el investigador deba despertar su agudeza e intuición para la reflexión (Heras Montoya, 1997; Rodríguez, Gil y García, 1996; Álvarez, 2008).

La principal característica del método es la participación del etnógrafo en la vida diaria de la comunidad durante un periodo de tiempo. Observando, escuchando e indagando cualquier tipo de información, hará uso de diferentes estrategias de investigación que lo conduzcan, en la medida de lo posible, a la reconstrucción de la cultura estudiada (Hammersley y Atkinson, 2005; Goetz y Le Compte, 1988). Estas estrategias son técnicas como la observación directa, las entrevistas formales e informales, las grabaciones auditivas, fotografías, videos y todo aquello que resulte de gran utilidad en el análisis y la interpretación del grupo estudiado (Nolla, 2007 y Álvarez, 2008). El proceso etnográfico no puede concluirse sin un informe detallado de la realidad observada; un documento donde se describan los actos presenciados, la información recogida, para así comprender lo que sucede. Por lo tanto, la elaboración del informe como producto textual es una etapa imprescindible en el modelo etnográfico (Velasco y Díaz de Rada, 2006; Pulido, 2003), de tal manera que tanto al trabajo de campo como la redacción del informe comprenden las dos fases de la etnografía.

La descripción reflexiva resultado de la experiencia etnográfica permite un análisis que relaciona a los individuos con su entorno social, económico, físico y simbólico de modo integral. Sin embargo, aunque no todo en una descripción pormenorizada es importante para una investigación concreta, el etnógrafo debe destacar lo que le parezca más significativo para contextualizar su caso de estudio. En el caso de la evaluación del contexto educativo, por ejemplo, la investigación etnográfica resulta una metodología muy adecuada para mejorar la transmisión y generación de conocimiento, así como para el análisis de la práctica docente. Al ser la educación una actividad plenamente inserta en la sociedad, ésta recibe una importante influencia de elementos exteriores a la escuela, entendiéndose estos factores como la familia, así como la comunidad en general, de tal manera que no puede decirse que se circunscribe únicamente al ámbito puramente escolar (Maturana y Garzón Daza, 2015; Álvarez, 2008; Serra, 2004; Wax y Wax, 1971; Spindler, 1982; Ogbu, 1989). Por esta razón, la etnografía contiene una importante potencialidad. Al ser una intervención pedagógica, en tanto que los profesores son parte del grupo e interactúan con sus estudiantes, es posible aprovechar la oportunidad de convertirlos en destacados observadores y entrevistadores de su entorno (Rockwell, 1994 y Parra, 1998).

Una de las características de esta etnografía educativa es la posibilidad de participación prolongada del investigador en el contexto a estudiar. Es decir, el cúmulo de experiencias y apreciaciones de orden cualitativo producto de las propias prácticas docentes son susceptibles de ser identificadas y exploradas para una mejora en la calidad didáctica. Al ser las escuelas expresiones concretas de la relación entre educación y sociedad, la etnografía educativa ofrece la oportunidad de identificar la diversidad, los comportamientos, el lenguaje, los estilo de aprender, la forma de pensar y todo aquello que se entiende como el capital cultural y social de una comunidad, así como también ponderar la respuesta de los alumnos ante las normas y prác- 
ticas establecidas por la cultura escolar, buscando de esta manera soluciones precisas a problemas reales de la sociedad (Carr y Kemmis, 1988; Montero-Sieburthes, 2005).

Dado que, actualmente, la educación formal forma parte fundamental del proceso cultural prolongado mediante el cual el ser humano aprende a integrarse en sociedad, la investigación etnográfica resulta un instrumento conveniente para la descripción, interpretación y comprensión de los contextos, así como para la transmisión de los resultados para el desarrollo de la realidad del entorno (Álvarez, 2008; Nolla, 1997). Desde este escenario, el docente emprende un análisis de carácter humanista, lo mismo fuera que dentro del contexto escolar. Esta circunstancia le ofrece la posibilidad de confrontar fenómenos desde los diferentes contextos en los que se producen, otorgándole a la investigación significados más trascendentales en el sentido social. Permite igualmente fomentar el diálogo en situaciones críticas, cotejar la realidad aparentemente homogénea e identificar fenómenos sociales, e incluso interculturales, dando oportunidad al docente de ejercer como un intelectual comprometido con la transformación social (Giroux, 1998 y Montero-Sieburthes, 2005).

\section{La etnografía aplicada a la innovación docente en el Grado de Turismo y Ocio}

Tal y como se ha señalado con anterioridad, la metodología etnográfica permite al docente, debido precisamente a su posición en la institucionalidad educativa, aprovechar tal circunstancia para recoger, analizar y proponer información útil de cara a la elaboración de nuevas estrategias educativas. En presente texto obedece a una investigación en curso llevada a cabo en el marco del Grado en Turismo y Ocio que oferta la Escuela Universitaria de Turismo Ostelea, centro adscrito a la Universitat de Lleida (UdL). En concreto, se analiza el uso de la etnografía dentro de las materias de "Desarrollo y Sociología de la Civilización del Ocio" y "Introducción a la Sociología y Psicología del Turismo", las cuales se encuentran enclavadas, en el Plan del Grado, en el segundo curso del mismo. Aunque los relatos que se ofrecen a continuación son descripciones detalladas de dos actividades concretas, enmarcables perfectamente en el espacio y en el tiempo, ambas cuentan con la experiencia atesorada por los docentes tras tres años de aplicación, es decir, en cierta manera suponen el resultado, más o menos acumulado, de la aplicación de esta metodología entre los años 2016 a 2018

\section{Desarrollo y Sociología de la Civilización del Ocio}

El objetivo general que se persigue con esta metodología en la asignatura "Desarrollo y Sociología de la Civilización del Ocio" es propiciar un acercamiento a diferentes manifestaciones del ocio en un entorno urbano, concretamente en la ciudad de Barcelona. Por este motivo, las sesiones son planificadas para ser llevadas a cabo durante un periodo de cuatro horas, de forma que supone una estancia en el campo de trabajo más prolongada de lo que supondría una clase ${ }^{3}$. Como se ha señalado, esta didáctica se ha realizado hasta en tres ocasiones, en diferentes cursos y años, con mínimas variaciones para cada caso. La experiencia de la mayoría de los estudiantes ha comportado un descubrimiento de parte de un barrio de Barcelona que, hasta el momento, sólo era conocido a través de referencias.

La actividad en sí, como parte de la asignatura reseñada, recibe el nombre de "Análisis de experiencias de ocio a lo largo de la vida" y tuvo lugar el 14 de marzo de 2018 en cinco plazas del barrio del Raval. Las plazas seleccionadas fueron: Terenci Moix, dels Àngels, Vicenç Martorell, Salvador Seguí y Sant Agustí, debido a los diferentes perfiles de personas y actividades que pueden encontrarse en cada una de ellas. La selección de estas plazas, así como del propio barrio del Raval, responde a un conocimiento profundo del mismo por parte de la profesora encargada ${ }^{4}$ de la asignatura, así como al hecho de que se encuentra íntimamente vinculado a la imagen de Barcelona como destino turístico de carácter cultural, además de como espacio especializado en actividades de ocio nocturno 5 .

La organización del trabajo de campo se realizó en tres fases: primero, la docente realizó una explicación de la actividad a todos los estudiantes. En ese momento, se constituyeron cinco grupos de trabajo, de entre cuatro y seis estudiantes y a cada uno se le asignó una plaza determinada. Segundo, cada grupo procedió al análisis de las experiencias de ocio en el lugar correspondiente. Tercero, se trabajaron las conclusiones de la actividad con todos los estudiantes. Una vez concluida la sesión, cada grupo de trabajo debía elaborar un informe en el que sistematizaran los resultados obtenidos y adjuntarlo al campus virtual de la Escuela en el plazo de una semana. Finalmente, a los estudiantes se les presentó un cuestionario para que evaluaran el uso de técnicas propias de la etnografía, tales como la observación, la realización de entrevistas y el desarrollo de un diario de campo, en el proceso de enseñanza-aprendizaje.

3 De forma ordinaria, las clases tienen una duración máxima de 2 horas.

4 Se trata de la Profesora Dra. Sheila Sánchez Bergara, vecina del barrio.

5 Para más información sobre el Raval ver Quaglieri, 2017. 


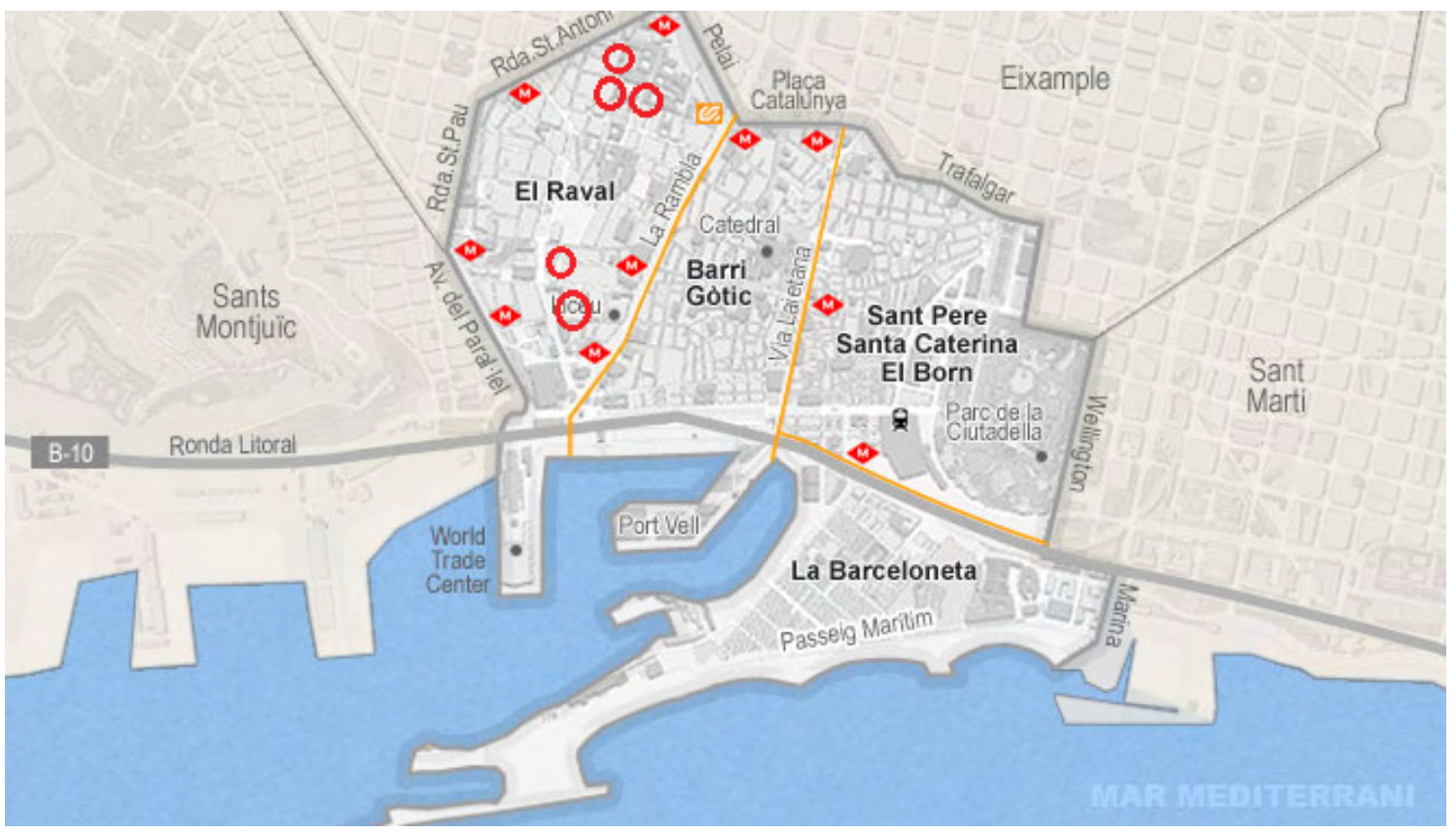

Mapa 1.- Localización de las Plazas estudiadas en el barrio del Raval Fuente: Elaboración propia a partir del Ajuntament de Barcelona

El 14 de marzo, la sesión se inició a las 9:00 horas y terminó a las 13:30. La idea era que los estudiantes pudieran ver el despertar del barrio e identificar variaciones en los usos de las plazas. Durante los primeros quince minutos de la sesión, la profesora presentó los objetivos que debían alcanzarse en cada una de las plazas; cómo debían desarrollar la actividad y con qué tiempo contaban para cada una de las fases. Con el objetivo de que el trabajo de campo acercara lo más posible a los estudiantes a las experiencias que debían analizar, se les indicó que dedicaran una primera parte del tiempo únicamente a observar el lugar y lo que en el mismo acontecía. Para ello, podían elegir libremente en qué sitio ubicarse en el espacio designado. A partir de ese primer contacto y teniendo en cuenta el contenido de la sesión precedente, debían preparar algunas preguntas para indagar sobre las experiencias de ocio a lo largo de la vida -itinerarios de ocio-, de al menos diez personas, a través de la observación y entrevistas informales. Además, se les animó a que tomaran fotos, vídeos y notas durante todo el tiempo que permanecieran en la plaza, para detectar los cambios y continuidades con el transcurso del tiempo.

A lo largo del trabajo de campo, los estudiantes debían recopilar información que les permitiera: a) Identificar y describir diferentes itinerarios de ocio; b) Describir los perfiles de las personas analizadas, y c) Identificar y describir elementos de los estilos de vida vinculados al ocio. En la última media hora de la sesión se realizó un debate en el que cada equipo presentó brevemente las cuestiones más significativas de su experiencia. En ese momento, los estudiantes contrastaron algunas de las diferencias existentes entre las plazas, tanto por las variaciones de edad de las personas que analizaron, como por el tipo de experiencias de ocio que realizaban en esos espacios.

Por ejemplo, los estudiantes detectaron que la Plaza Salvador Seguí es fundamentalmente un sitio de paso, con un equipamiento y mobiliario público -bancos, parques infantiles, etc.- limitado para desarrollar actividades prolongadas. A su vez, manifestaron que, con el avance de las horas, comenzaron a ocuparse las terrazas de los bares circundantes por personas de rangos de edad variada; identificaron a un grupo de estudiantes con su profesor que visitaban la filmoteca como parte de una actividad escolar; observaron a algunos adultos mayores que se sentaban en los bancos públicos a contemplar el lugar, pasar el rato y tomar el sol; advirtieron un número significativo de adultos jóvenes paseando con sus perros y, por último, detectaron actividades de prostitución en una de las calles aledañas. Estos resultados contrastaron significativamente con los obtenidos por los grupos de la Plaza Terenci Moix y de la Plaza dels Àngels. En la primera, la principal actividad de ocio detectada fue el baloncesto, llevada a cabo por un grupo de adultos jóvenes de manera regular en ese espacio, sin duda debido a los equipamientos existentes en la zona. Mientras que, en la segunda, primaba la práctica del skateboard por jóvenes y adultos jóvenes que, de manera ocasional y/o regular, utilizan las características del espacio -una explanada amplia y lisa- para ello. 


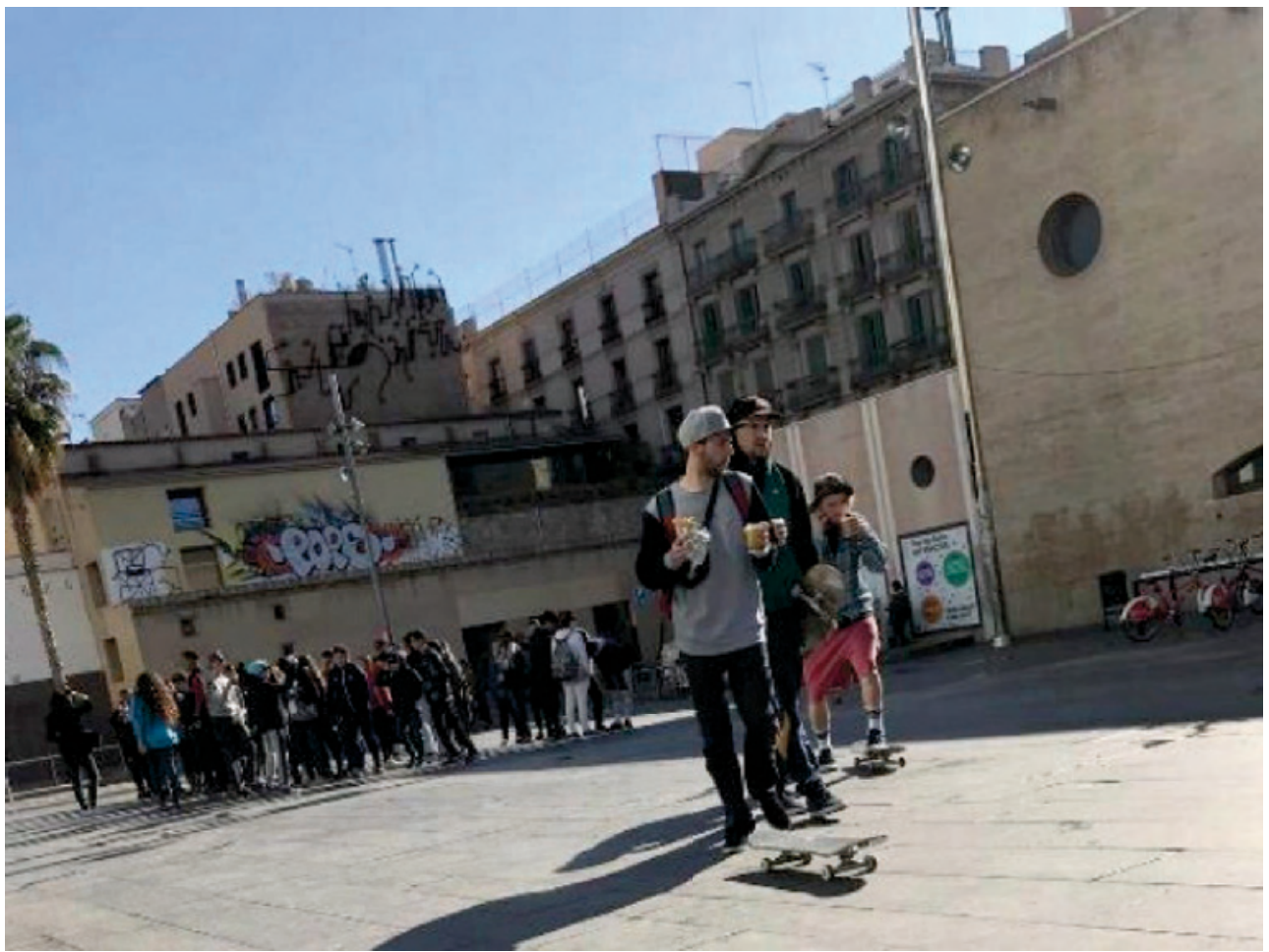

Foto 1.- Un momento del trabajo de campo llevado a cabo en la Plaça dels Àngels (14/03/2017) Fuente: Equipo Plaça dels Àngels

El debate colectivo realizado al final de la sesión permitió que cada grupo resumiera y diera a conocer sus principales resultados, comparándolos, a su vez, con los de sus compañeros. Asimismo, la profesora los invitó a visitar sus respectivas plazas en un horario diferente, por ejemplo, al final de la tarde, en la noche y/o en el fin de semana. El objetivo perseguido con esta recomendación era que los estudiantes profundizaran en el estudio de los conceptos indicados y los espacios asignados, al tiempo que ampliaran los primeros resultados obtenidos.

Una vez evaluados los informes presentados por cada grupo, se evidenció que, en general, las fotografías, la observación y las entrevistas realizadas en cada plaza contribuyeron al análisis realizado por cada grupo y dieron soporte a los trabajos presentados. Además, la organización de los resultados empíricos se ajustó a los conceptos teóricos previamente estudiados en clase. Con lo cual, se evidenció un desarrollo de la capacidad de describir y analizar situaciones prácticas a través de conceptos, en este caso concreto, itinerarios de ocio y estilos de vida de ocio. Cabe resaltar, además, que a los estudiantes se les realizó un cuestionario para que evaluaran la idoneidad de este trabajo de campo en el proceso de enseñanza-aprendizaje. Las opiniones fueron mayoritariamente positivas, no obstante, en los comentarios refirieron la necesidad de contar también con otras didácticas, tales como, sesiones teórico-explicativas y tutorías personalizadas que les permitan reforzar los conocimientos y aclarar las dudas.

\section{Introducción a la Sociología y Psicología del Turismo}

La asignatura de "Introducción a la Sociología y Psicología del Turismo" pretende ofrecer a los estudiantes la oportunidad de dotarse de una serie de herramientas metodológicas y teóricas que les permita observar el turismo no sólo como un sector económico, sino también como elemento central de las sociedades occidentales contemporáneas. La aproximación que se realiza al turismo es, por un lado, como medio y, por otro, como manifestación del proceso de globalización, esto es, como auténtico vehículo de transformación de la vida y las experiencias de los grupos sociales partícipes. La asignatura cuenta con una parte mayoritaria dedicada al aprendizaje de referencias y marcos teóricos en el aula, pero también de determinadas sesiones de trabajo de campo: actividades en el exterior dedicadas a la realización de acercamientos etnográficos a realidades cercanas. El tiempo dedicado a éstas excede la duración de una sesión normal de 2 horas, extendiéndose hasta las 5. La dinámica detallada se ha realizado hasta en 3 ocasiones desde el inicio del Grado. 


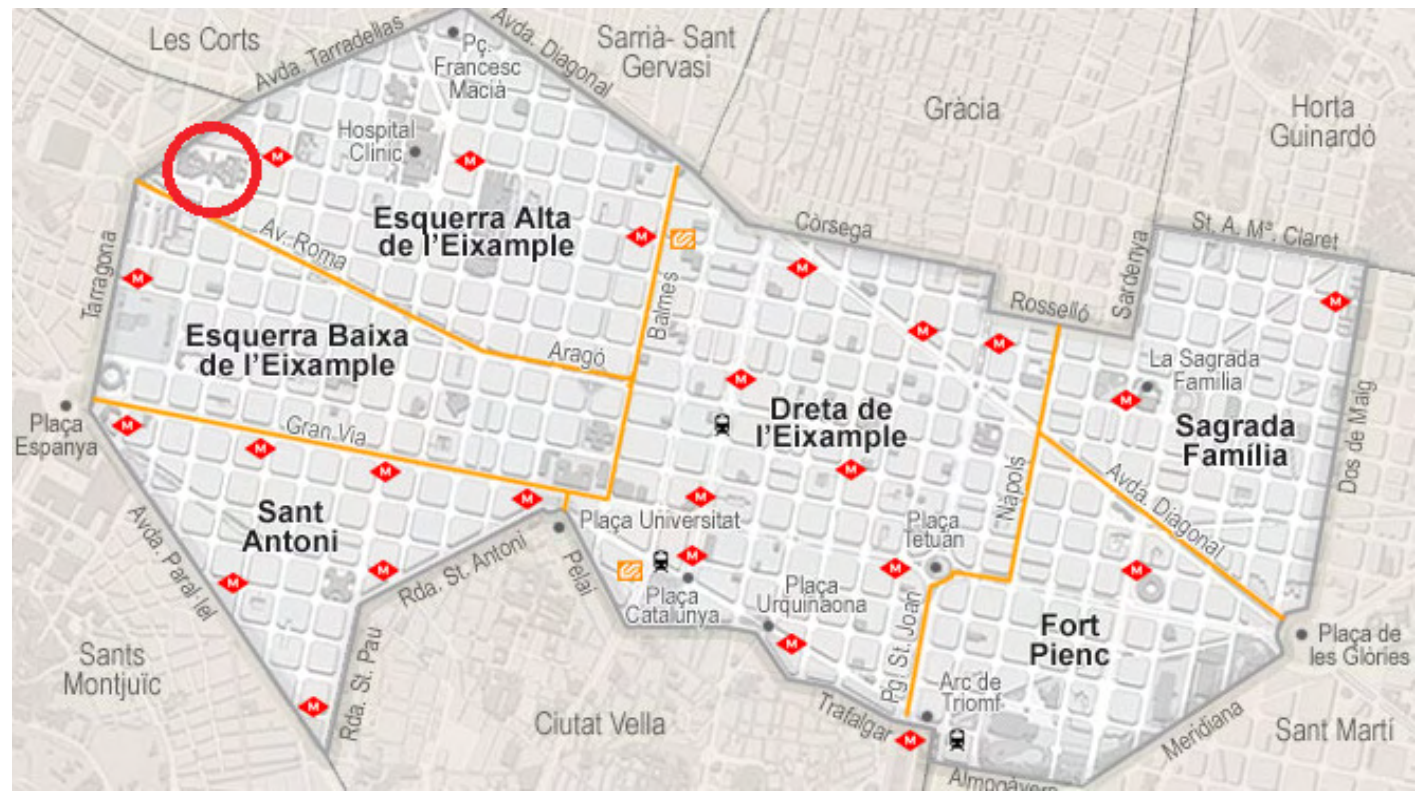

\section{Mapa 2.- Localización de la Cárcel Modelo en el Eixample Esquerra Fuente: Elaboración propia a partir del Ajuntament de Barcelona}

La siguiente descripción se corresponde con la sesión llevada a cabo el día 17 de octubre de 2018 en horario de 14.00 a $19.00 \mathrm{~h}^{-}{ }^{6}$. Ésta estuvo centrada en el proceso de transformación que está viviendo el área inmediata a la Cárcel Modelo ${ }^{7}$, en el barcelonés barrio del Eixample, concretamente la establecida por el marco de las calles Entença, Nicaragua, Roselló y Provença. El Ayuntamiento de la ciudad lleva tiempo barajando la posibilidad de abrir, en las antiguas instalaciones carcelarias, un espacio dedicado a la memoria de carácter museístico. Durante el tiempo que se han llevado a cabo las actividades docentes vinculadas a la etnografía, se han visto escasos avances en el desarrollo del proyecto, más allá de la organización de una serie de visitas guiadas o la apertura parcial al público del primer patio del recinto. Sin embargo, es evidente el efecto producido en el entorno tras el anuncio institucional del cambio de uso del parcelario afectado. La depresión urbana ocasionada por la presencia continuada de la cárcel durante más de cien años ha dado paso a importantes movimientos especulativos en la zona, como la amenaza y posterior desahucio vivido por los inquilinos de la finca $\mathrm{n}^{\circ} 151$ de la calle Entença ${ }^{8}$, o la renovación parcial de su entramado comercial y de restauración. En esta ocasión, la selección del emplazamiento a estudiar se debió a un motivo doble: la cercanía a la sede principal de la Escuela Universitaria de Turismo en la calle Aragó, y la posibilidad que ofrecía estudiar in situ el impacto generado, desde su inicio, de la apertura de un nuevo recurso turístico para la ciudad.

La actividad docente estuvo organizada en torno a 4 momentos diferentes: al inicio de la misma, los estudiantes asistieron en el aula a una explicación detallada y completa sobre el desarrollo de la salida (objetivos, situación actual de la zona, cronograma de la actividad, etc.); posteriormente se realizó la salida de campo, la cual contó, de forma permanente, con la asistencia del profesor al cargo de la asignatura; una vez de vuelta a la clase, se procedió a debatir y consensuar las principales características que ofrecía el proceso estudiado, para lo que se utilizó una matriz DAFO (Debilidades, Amenazas, Fortalezas, Oportunidades); para finalizar, los alumnos y alumnas, divididas en grupos de 4-5 miembros, consensuaron las líneas estratégicas y los proyectos concretos que podrían llevarse a cabo para poner en marcha el museo a la vez que se minimiza el impacto en la zona.

6 Se trató, en esta ocasión, de una sesión conjunta con los estudiantes de la Materia Transversal “Antropología del Turismo". Ambas son impartidas por el Profesor Dr. José A. Mansilla.

7 La Cárcel Modelo de Barcelona fue vaciada de presos y cerrada por la Generalitat de Catalunya el pasado mes de mayo de 2017. El cierre se produjo en el marco de un acuerdo con el Ayuntamiento de Barcelona por el cual la institución municipal se comprometía a ceder los terrenos para construir un nuevo recinto carcelario a cambio de ceder el suelo urbano que liberaría la Modelo. Entre las ideas barajadas para el espacio que deja la antigua prisión -dos manzanas del Eixample- se encuentra, además de una Escuela y un Hogar para la Tercera Edad y una dotación de Vivienda Protegida, la instalación de un Museo dedicado a la memoria de los presos que por ella pasaron. Para más información ver "La Modelo echa el cierre", El País, 08/06/2017 y "La Modelo abre sus puertas para siempre", El País, 22/01/2018.

8 Para más información ver "Entença 151, un edificio amenazado", El Periódico, 30/05/2017 o "Tenso y accidentado desalojo en Entença 151”, Metrópolis Abierta, 08/08/2017. 
Llegado el día de la salida, se procedió, inicialmente, a repartir entre el alumnado una breve ficha con información básica del recinto de la Modelo: plano, localización, información recogida en prensa, etc. La introducción a la actividad y la explicación sobre el proyecto municipal para la zona supuso un total de, aproximadamente, una hora trabajo docente ${ }^{9}$. La idea era que pasaran en torno a 2 horas en las inmediaciones de la Cárcel y recogieran la mayor cantidad de información posible a través de las herramientas que facilita la etnografía -no solo la observación-participante, sino también el establecimiento de diálogos informales, o incluso alguna pequeña entrevista, con aquellas personas que se encontraran en el entorno y pudieran suministrar datos-, además de la toma de notas, fotografías o pequeños dibujos o croquis de las inmediaciones. En este sentido, se le dio especial importancia al acopio de la opinión parte de los vecinos y vecinas del área sobre las intenciones del Ayuntamiento para el futuro espacio liberado.

Durante las dos horas que duraba esta parte del ejercicio, los estudiantes debían intentar poner en marcha su "imaginación sociológica" (Giddens, 1992), de forma que pudieran identificar las características actuales del área, no sólo a nivel físico-urbanístico, sino, sobre todo y principalmente, de los distintos perfiles de las personas que se encontraran, y, además, prever los posibles impactos que tanto el desarrollo de las obras como el funcionamiento ordinario del futuro museo ocasionara para el vecindario.

Así, a modo de ejemplo, los estudiantes mantuvieron dos conversaciones con comerciantes de la zona. En la primera de ellas, llevada a cabo al dueño de una carnicería rumana ${ }^{10}$ de la esquina de Entença con Provença, éstos se interesaron por su opinión con respecto a los cambios más relevantes vividos por la zona desde el cierre de la prisión. El propietario del establecimiento les hizo ver que algunas tiendas y bares locales, fundamentalmente aquellos que ofrecían productos y servicios a los funcionarios de la cárcel, no habían visto con muy buenos ojos su cierre. Además, manifestó un profundo desconocimiento sobre la fecha de inicio de las obras o de su posible duración. En el segundo de los diálogos, protagonizado, en esta ocasión, por una farmacéutica de la esquina entre las calles Provença y Vilamarí, los estudiantes fueron informados del cierto descrédito que el proceso participativo emprendido por el Ayuntamiento estaba teniendo entre algunos de los vecinos y vecinas de la zona, así como por parte de los pequeñas empresarios del barrio. Los continuos cambios de planes y fechas de inicio de las obras, así como los encuentros llevados a cabo entre los técnicos y políticos municipales junto al vecindario, habían acabado trasladando una imagen de inutilidad y pérdida de tiempo, desvalorizando la capacidad de incidencia final de los participantes.

Una vez reunidos de nuevo en el aula, se elaboró, de manera abierta, una amplia matriz DAFO que permitió exponer los principales elementos recogidos durante la actividad de calle. Esto permitió agrupar bajo etiquetas altamente significativas los elementos fundamentales de la actividad. El objetivo fue, sobre todo, contar con una imagen -el DAFO permaneció visible en todo momento en la pizarra del aula- más o menos completa de la información recogida, de forma que fuera posible plantear las líneas de actuación que protagonizarían la siguiente fase de la actividad.

Para finalizar, los estudiantes, divididos en pequeños grupos, se reunieron de forma apartada para, así, elaborar una serie de propuestas estratégicas generales de las cuales colgarían, a su vez, proyectos concretos. El objetivo era, por un lado, canalizar y hacer posible la iniciativa del Ayuntamiento de poner en marcha un museo dedicado a la memoria de la Modelo y, por otro, establecer todas las medidas posibles que evitaran impactos negativos para el tejido social y económico de la zona, como los ya mencionados procesos especulativos o el desplazamiento del comercio de proximidad. Esto permitió a los alumnos y alumnas comprender la realidad de un área concreta y experimentar su capacidad de articular propuestas plausibles para un proyecto real.

Una vez acabada la actividad, se mantuvo un pequeño debate sobre la idoneidad de llevar a cabo una actividad de estas características, hecho que permitió recoger unas opiniones altamente positivas al respecto.

\section{A modo de conclusión}

La necesidad creciente de adaptar la educación al compás de los tiempos, proceso éste, además, impulsado a nivel español por una normativa que apuesta por considerar la educación como un proceso que abarca la totalidad de la vida de los individuos, impulsa a los docentes y a las instituciones educativas a apostar fuertemente por diversas iniciativas de innovación docente. En este sentido, metodologías inicialmente ajenas a las dinámicas formativas tradicionales, como la etnografía, herramienta fundamental de las ciencias sociales, se presentan como oportunidades ideales para ampliar, de forma crítica, la relación de los alumnos y alumnas con el profesorado, así como con el contexto en el que ésta se desarrolla.

9 Con anterioridad, a lo largo del año 2009, el Ayuntamiento y el vecindario ya había emprendido un proceso participativo para decidir qué hacer en la zona una vez eliminada la cárcel. Para más información ver: https://presomodel.wordpress.com/

10 En la parte de la calle Provença que discurre paralela a los muros de la cárcel existen numerosos establecimientos regentados por población rumana. 


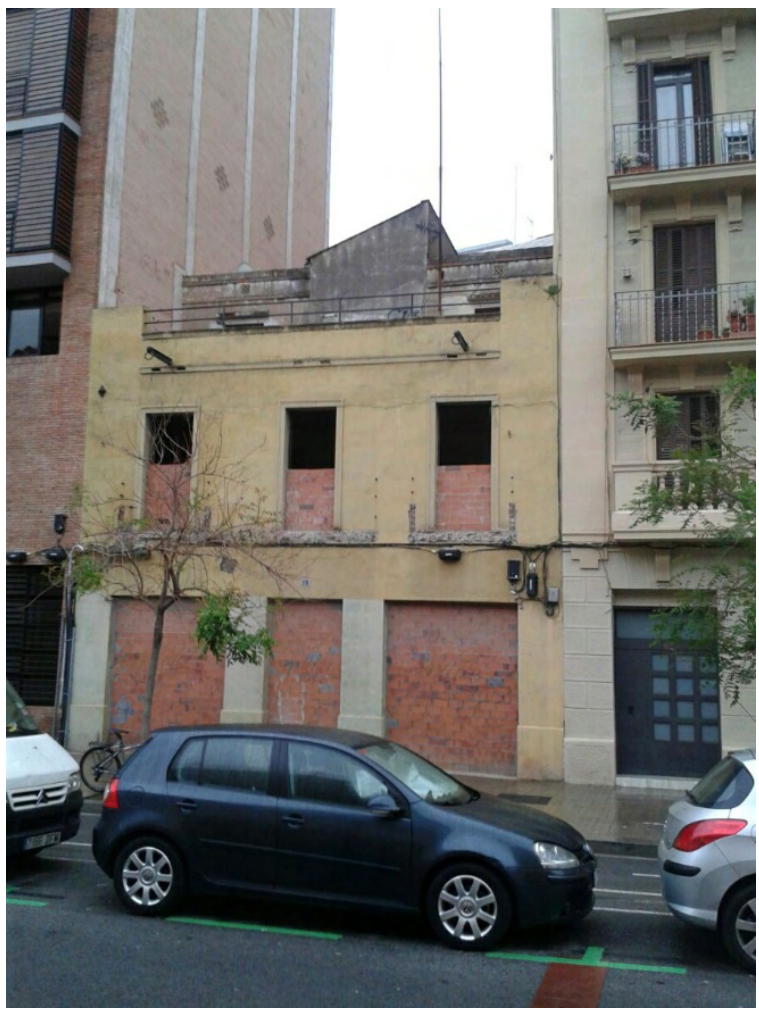

Foto 2.- Obras abandonadas en la calle Provença que manifiestan la denominada depresión urbana $(26 / 04 / 2017)$

Fuente: José A. Mansilla

El caso aquí expuesto, la aplicación de la etnografía al estudio de zonas singulares de la ciudad de Barcelona, llevado a cabo en el marco de las asignaturas de "Desarrollo y Sociología de la Civilización del Ocio" e "Introducción a la Sociología y Psicología del Turismo" del Grado de Turismo y Ocio, forma parte de una investigación en marcha que persigue analizar cómo esta metodología permite al alumnado visibilizar fehacientemente la relación entre los resultados empíricos obtenidos durante el desarrollo de los ejercicios y conceptos teóricos previamente vistos en clase. Tanto la visita a las distintas plazas del barrio del Raval, como el análisis del impacto que la transformación de la Cárcel Modelo ha tenido y tendrá en su entorno más inmediato, facultan a profesores y estudiantes, además, a tomar consciencia de la aplicación directa de sus estudios a la vida real, así como a prever la potencialidad del Grado estudiado.

La experiencia vivida tras el despliegue de esta metodología podría permitir en un futuro introducir la práctica etnográfica en un mayor número de asignaturas e, incluso, elaborar un material pedagógico específico destinado a futuros estudiantes de turismo y ocio. Quedarían pendientes dos tareas potenciales: por un lado, continuar profundizando en la aplicación de la etnografía a través de la realización de este tipo de actividades en cursos venideros y espacios distintos, y, por otro, examinar la posibilidad de aplicar el método etnográfico al estudio de los propios docentes mediante actividades de evaluación recíproca estudiantesprofesor. En definitiva, el uso de la metodología etnográfica en la educación avanzaría, no solo, el establecimiento y expansión de nuevas herramientas formativas, sino también asegurar un futuro prometedor a un método de investigación que surgió en un contexto y un tiempo muy distintos.

\section{Bibliografía}

Aguirre Baztán, A. (1995). Etnografía. Metodología cualitativa en la investigación sociocultural. Barcelona, Marcombo.

Álvarez Álvarez, C. (2008). La etnografía como modelo de investigación en educación. Gazeta de Antropología, 24 (1), artículo 10. Recuperado de http://hdl.handle.net/10481/6998

Carr, W. y Kemmis, S. (1988). Teoría crítica de la enseñanza. La investigación acción en la formación del profesorado. Barcelona: Martínez Roca.

Giddens, A. (1992). Sociología. Madrid: Alianza Editorial.

Giroux, H. y Mclaren, P. (1998). Sociedad, cultura y educación. Madrid: Miño y Dávila Editores. 
Goetz, J. P., Lecompte, M. D. (1988). Etnografia y diseño cualitativo en investigación educativa. Madrid: Morata.

Hammersley, M. y P. Atkinson (2005). Etnografía. Métodos de investigación. Barcelona: Paidós.

Heras Montoya, L. (1997). Comprender el espacio educativo. Investigación etnográfica sobre un centro escolar. Málaga: Aljibe.

Malinowski, B. (1986). Los argonautas del Pacífico Occidental. Barcelona: Planeta - De Agostini.

Maturana Moreno, G. A. y Garzón Daza, C. (2015). La etnografía en el ámbito educativo: una alternativa metodológica de investigación al servicio docente. Revista de Educación y Desarrollo Social, 9(2), 192-205.

Mauss, M. (1974). Introducción a la etnografía. Madrid: Istmo.

Montero-Sieburth, M., Pozo Lorente, T. y Cabrera Pérez, L. (2005). Aportaciones de la Autoetnografía a la investigación intercultural: Un Análisis Crítico de un Centro Educativo de la Ciudad de Boston, Massachussets. En Soriano Ayala, C. (ed.), Calidad Educativa en la Escuela Intercultural (pp. 389-396). Almería: Universidad de Almería.

Nolla Cao, N. (1997). Etnografía: una alternativa más en la investigación pedagógica. Revista Cubana de Educación Media y Superior, 11: 107-115.

Ogbu, J. U. (1989). Antropología de la educación. En Husen, T. y Neville Postelthwaite, T. (Dir.), Enciclopedia Internacional de la Educación. Madrid, Vicens Vives/MEC.

Pulido, R. A. (2003). Una visión sobre la etnografía educativa a través del caso de unos alumnos que se burlaron del etnógrafo que sustituía a la maestra. Revista Ágora Digital, 6. Recuperado de http:// hdl.handle.net/10272/3520.

Quaglieri, A. y Scarnato, A. (2017). The Barrio Chino as last frontier: the penetration of everyday tourism in the dodgy heart of the Raval. En Gravari-Barbas, M. y Guinand, S. (coord.) Tourism and Gentrification in Contemporary Metropolises (pp. 121-147). Oxford: Routledge.

Rodríguez Gómez, G., Gil Flores J. y García Jiménez, E. (1996). Metodología de la investigación cualitativa. Málaga: Aljibe.

Sanchiz Ochoa, P. y Cantón Delgado, M. (1995). Acceso y adaptación al campo. En Aguirre Baztán, A. (coord.), Etnografía. Metodología cualitativa en la investigación sociocultural (pp. 128-134). Barcelona, Marcombo.

Serra, C. (2004). Etnografía escolar, etnografía de la educación. Revista de Educación, 334: 165-176.

Spindler, G. D. (1982). Doing Ethnography of Schooling: Educational Anthropology in Action. New York: Holt, Rinehart y Winston

Velasco, H. y Á. Díaz de Rada (2006). La lógica de la investigación etnográfica. Un modelo de trabajo para etnógrafos de escuela. Madrid: Trotta.

Wax, M. y Wax, R.: (1971). Great Tradition, Little Tradition, and Formal Education. En Wax, M., Diamond, S. y Gearing, F. (Eds.). Anthropological Perspectives on Education (pp. 3-27). New York, Basic Books, 1971. 